\title{
INCREASING POWERS IN A DEGENERATE PARABOLIC LOGISTIC EQUATION
}

\author{
JOSÉ FRANCISCO RODRIGUES AND HUGO TAVARES
}

\begin{abstract}
The purpose of this paper is to study the asymptotic behavior of the positive solutions of the problem$$
\partial_{t} u-\Delta u=a u-b(x) u^{p} \text { in } \Omega \times \mathbb{R}^{+}, u(0)=u_{0},\left.u(t)\right|_{\partial \Omega}=0
$$

as $p \rightarrow+\infty$, where $\Omega$ is a bounded domain and $b(x)$ is a nonnegative function. We deduce that the limiting configuration solves a parabolic obstacle problem, and afterwards we fully describe its long time behavior.
\end{abstract}

\section{INTRODUCTION}

In this paper we are interested in the study of the parabolic problem

$$
\left\{\begin{aligned}
\partial_{t} u-\Delta u & =a u-b(x) u^{p} & & \text { in } Q:=\Omega \times(0,+\infty) \\
u & =0 & & \text { on } \partial \Omega \times(0,+\infty) \\
u(0) & =u_{0} & & \text { in } \Omega
\end{aligned}\right.
$$

where $a>0, p>1, b \in L^{\infty}(\Omega)$ is a nonnegative function and $\Omega$ is a bounded domain with smooth boundary. Such system arises in population dynamics, where $u$ denotes the population density of a given specie, subject to a logistic-type law.

It is well known that under these assumptions and for very general $u_{0}$ 's, (1) admits a unique global positive solution $u_{p}=u_{p}(x, t)$. In fact, in order to deduce the existence result, one can make the change of variables $v=e^{-a t} u$, and deduce that $v$ satisfies $\partial_{t} v-\Delta v+b(x) e^{p a t} v^{p}=0$. As $v \mapsto b(x) e^{p a t}|v|^{p-1} v$ is monotone nondecreasing, the theory of monotone operators (cf. [14, 16]) immediately provides existence of solution for the problem in $v$, and hence also for (10).

One of our main interests is the study of the solution $u_{p}$ as $p \rightarrow+\infty$. As we will see, in the limit we will obtain a parabolic obstacle problem, and afterwards we fully describe its asymptotic limit as $t \rightarrow+\infty$.

This study is mainly inspired by the works of Dancer et al 4, 5, 6, where the stationary version of (1) is addressed. Let us describe in detail their results. Consider the elliptic problem

$$
-\Delta u=a u-b(x) u^{p}, \quad u \in H_{0}^{1}(\Omega)
$$

and, for each domain $\omega \subseteq \mathbb{R}^{N}$, denote by $\lambda_{1}(\omega)$ the first eigenvalue of $-\Delta$ in $H_{0}^{1}(\omega)$. Assuming $b \in C(\bar{\Omega})$, the study is divided in two cases: the so called nondegenerate

Date: May 21, 2012.

2010 Mathematics Subject Classification. Primary: 35B40 ; Secondary: 35B09, 35K91.

Key words and phrases. parabolic logistic equation, obstacle problem, positive solutions, increasing powers, sub and supersolutions.

Both authors were supported by Fundação para a Ciência e a Tecnologia (FCT), PEst OE/MAT/UI0209/2011.

The second author was also supported by the FCT grant SFRH/BPD/69314/201. 
case (where $\min _{\bar{\Omega}} b(x)>0$ ) and the degenerate one (where $\Omega_{0}:=\operatorname{int}\{x \in \Omega$ : $b(x)=0\} \neq \emptyset$ and has smooth boundary).

In the nondegenerate case, it is standard to check (see for instance [8, Lemma $3.1 \&$ Theorem 3.5]) that (2) has a positive solution if and only if $a>\lambda_{1}(\Omega)$. For each $a>\lambda_{1}(\Omega)$ fixed, then in [5] it is shown that $u_{p} \rightarrow w$ in $C^{1}(\bar{\Omega})$ as $p \rightarrow+\infty$, where $w$ is the unique solution of the obstacle-type problem

$$
-\Delta w=a w \chi_{\{w<1\}}, \quad w>0,\left.\quad w\right|_{\partial \Omega}=0,\|w\|_{\infty}=1 .
$$

It is observed in 4 that $u$ is also the unique positive solution of the variational inequality

$$
w \in \mathbb{K}: \quad \int_{\Omega} \nabla w \cdot \nabla(v-w) d x \geqslant \int_{\Omega} a w(v-w) d x, \quad \forall v \in \mathbb{K},
$$

where

$$
\mathbb{K}=\left\{w \in H_{0}^{1}(\Omega): w \leqslant 1 \text { a.e. in } \Omega\right\} .
$$

In the degenerate case, on the other hand, problem (2) has a positive solution if and only if $a \in\left(\lambda_{1}(\Omega), \lambda_{1}\left(\Omega_{0}\right)\right)$. For such $a$ 's, assuming that $\Omega_{0} \Subset \Omega$, if we combine the results in [5, 6], we see that $u_{p} \rightarrow w$ in $L^{q}(\Omega)$ for every $q \geqslant 1$, where now $w$ is the unique nontrivial nonnegative solution of

$$
v \in \mathbb{K}_{0}: \quad \int_{\Omega} \nabla w \cdot \nabla(v-w) d x \geqslant \int_{\Omega} a w(v-w) d x, \quad \forall v \in \mathbb{K}_{0},
$$

with

$$
\mathbb{K}_{0}=\left\{w \in H_{0}^{1}(\Omega): w \leqslant 1 \text { a.e. in } \Omega \backslash \Omega_{0}\right\} .
$$

The uniqueness result was the subject of the paper [6]. Therefore, whenever $b(x) \neq$ 0 , the term $b(x) u^{p}$ strongly penalizes the points where $u_{p}>1$, forcing the limiting solution to be bellow the obstacle 1 at such points.

Our first aim is to extend these conclusions for the parabolic case (1). While doing this, our concern was also to relax some of the assumptions considered in previous papers, namely the continuity of $b$ as well as the condition of $\Omega_{0}$ being in the interior of $\Omega$. In view of that, consider the following conditions for $b$ :

(b1) $b \in L^{\infty}(\Omega)$;

(b2) there exists $\Omega_{0}$, an open domain with smooth boundary, such that

$$
b(x)=0 \text { a.e. on } \Omega_{0} \text {, and }
$$

$$
\forall \Omega^{\prime} \Subset \Omega \backslash \Omega_{0} \text { open } \exists \underline{b}>0 \text { such that } b(x) \geqslant \underline{b} \text { a.e. in } \Omega^{\prime} .
$$

Observe that in (b2) $\Omega_{0}=\emptyset$ is allowed, and $\bar{\Omega}_{0}$ may intersect $\partial \Omega$. Continuous functions with regular nodal sets or characteristic functions of open smooth domains are typical examples of functions satisfying (b1)-(b2). As for the initial data, we consider:

(H1) $u_{0} \in H_{0}^{1}(\Omega) \cap L^{\infty}(\Omega)$;

(H2) $0 \leqslant u_{0} \leqslant 1$ a.e. in $\Omega \backslash \Omega_{0}$.

Our first main result is the following.

Theorem 1.1. Assume that $b$ satisfies (b1)-(b2) and $u_{0}$ satisfies (H1)-(H2). Then there exists a function $u$ such that, given $T>0, u \in L^{\infty}\left(0, T ; H_{0}^{1}(\Omega)\right) \cap H^{1}\left(0, T ; L^{2}(\Omega)\right)$ and

$$
\begin{aligned}
& u_{p} \rightarrow u \quad \text { strongly in } L^{2}\left(0, T ; H_{0}^{1}(\Omega)\right) ; \\
& \partial_{t} u_{p} \rightarrow \partial_{t} u \quad \text { weakly in } L^{2}\left(Q_{T}\right) .
\end{aligned}
$$


Moreover $u$ is the unique solution of the following problem:

for a.e. $t>0, u(t) \in \mathbb{K}_{0}$ :

$\int_{\Omega} \partial_{t} u(t)(v-u(t)) d x+\int_{\Omega} \nabla u(t) \cdot \nabla(v-u(t)) d x \geqslant \int_{\Omega} a u(t)(v-u(t)) d x$

for every $v \in \mathbb{K}_{0}$, with the initial condition $u(0)=u_{0}$.

Next we turn to the long time behavior of the solution of (6).

Theorem 1.2. Suppose the $b$ satisfies (b1)-(b2) and take $u_{0}$ verifying (H1)-(H2). Fix $a \in\left(\lambda_{1}(\Omega), \lambda_{1}\left(\Omega_{0}\right)\right)$. Let $u$ be the unique positive solution of (6) and take $w$ the unique nontrivial nonnegative solution of (5). Then $\|w\|_{\infty}=1$ and

$$
u(t) \rightarrow w \quad \text { strongly in } H_{0}^{1}(\Omega) \text {, as } t \rightarrow+\infty \text {. }
$$

Moreover if $a<\lambda_{1}(\Omega)$ then $\|u(t)\|_{H_{0}^{1}(\Omega)} \rightarrow 0$, and if $a \geqslant \lambda_{1}\left(\Omega_{0}\right)$ then both $\|u(t)\|_{\infty}$ and $\|u(t)\|_{H_{0}^{1}(\Omega)}$ go to $+\infty$ as $t \rightarrow+\infty$.

We remark that in the case $\Omega_{0}=\emptyset$ we set $\lambda_{1}\left(\Omega_{0}\right):=+\infty$, and $a \geqslant \lambda_{1}\left(\Omega_{0}\right)$ is an empty condition. The case $a=\lambda_{1}(\Omega)$ is the subject of Remark 4.5 .

Under some stronger regularity assumptions on $b, u_{0}$ and $\Omega_{0}$, it is known (cf. [8. Theorem 3.7] or [7, Theorem 2.2]) that $u_{p}(t, x)$ converges to the unique positive solution of (2) whenever $a \in\left(\lambda_{1}(\Omega), \lambda_{1}\left(\Omega_{0}\right)\right)$. Hence in this situation, if we combine all this information together with the results obtained in this paper, then we can conclude that the following diagram commutes:

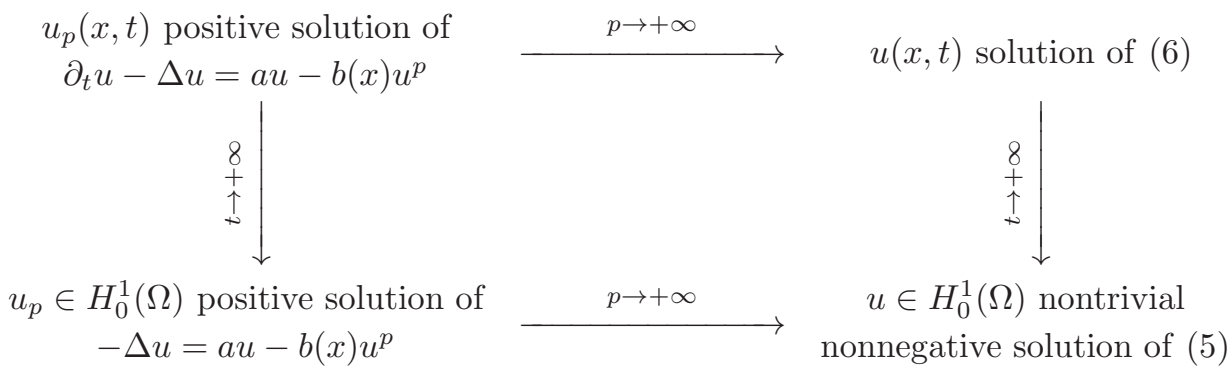

The proof of Theorem 1.1 uses a different approach with respect to the works by Dancer et al.. While in [5 the authors use fine properties of functions in Sobolev spaces, here we follow some of the ideas present in the works [1, 2], and show that a uniform bound on the quantity

$$
\iint_{Q_{T}} b(x) u_{p}^{p+1} d x d t \quad(\text { for each } T>0),
$$

implies that $u(t) \in \mathbb{K}_{0}$ for a.e. $t>0$ (see the key Lemma2.4 ahead). As for the proof of Theorem 1.2. the most difficult part is to show that when $a \in\left(\lambda_{1}(\Omega), \lambda_{1}\left(\Omega_{0}\right)\right)$, $u_{p}(x, t)$ does not go to the trivial solution of (5). The key point here is to construct a subsolution of (11) independent of $p$. It turns out that to do this one needs to get a more complete understanding of the nondegenerate case, and to have a stronger convergence of $u_{p}$ to $u$ as $p \rightarrow+\infty$. So we dedicate a part of this paper to the study of this case. To state the results, let us start by defining for each $0<t_{1}<t_{2}$ and $Q_{t_{1}, t_{2}}:=\Omega \times\left(t_{1}, t_{2}\right)$ the spaces $C_{\alpha}^{1,0}\left(\bar{Q}_{t_{1}, t_{2}}\right)$ and $W_{q}^{2,1}\left(Q_{t_{1}, t_{2}}\right)$. For $q \geqslant 1$, the 
space $W_{q}^{2,1}\left(Q_{t_{1}, t_{2}}\right)$ is the set of elements in $L^{q}\left(Q_{t_{1}, t_{2}}\right)$ with partial derivatives $\partial_{t} u$, $D_{x} u, D_{x}^{2} u$ in $L^{q}\left(Q_{t_{1}, t_{2}}\right)$. It is a Banach space equipped with the norm

$\|u\|_{2,1 ; q, Q_{t_{1}, t_{2}}}=\|u\|_{L^{q}\left(Q_{t_{1}, t_{2}}\right)}+\left\|D_{x} u\right\|_{L^{q}\left(Q_{t_{1}, t_{2}}\right)}+\left\|D_{x}^{2} u\right\|_{L^{q}\left(Q_{t_{1}, t_{2}}\right)}+\left\|\partial_{t} u\right\|_{L^{q}\left(Q_{t_{1}, t_{2}}\right)}$.

For each $\alpha \in(0,1), C_{\alpha}^{1,0}\left(\bar{Q}_{t_{1}, t_{2}}\right)$ is the space of Hölder functions $u$ in $\bar{Q}_{t_{1}, t_{2}}$ with exponent $\alpha$ in the $x$-variable and $\alpha / 2$ in the $t$-variable, with $D_{x} u$ satisfying the same property. More precisely, defining the Hölder semi-norm

$[u]_{\alpha, Q_{t_{1}, t_{2}}}:=\sup \left\{\frac{\left|u(x, t)-u\left(x^{\prime}, t^{\prime}\right)\right|}{\left|x-x^{\prime}\right|^{\alpha}+\left|t-t^{\prime}\right|^{\alpha / 2}}, \quad x, x^{\prime} \in \bar{\Omega}, t, t^{\prime} \in\left[t_{1}, t_{2}\right], \quad(x, t) \neq\left(x^{\prime}, t^{\prime}\right)\right\}$,

we have that

$$
\begin{aligned}
C_{\alpha}^{1,0}\left(\bar{Q}_{t_{1}, t_{2}}\right):=\left\{u:\|u\|_{C_{\alpha}^{1,0}\left(\bar{Q}_{t_{1}, t_{2}}\right)}:=\right. & \|u\|_{L^{\infty}\left(Q_{t_{1}, t_{2}}\right)}+\left\|D_{x} u\right\|_{L^{\infty}\left(Q_{t_{1}, t_{2}}\right)} \\
& \left.+[u]_{\alpha, Q_{t_{1}, t_{2}}}+\left[D_{x} u\right]_{\alpha, Q_{t_{1}, t_{2}}}<+\infty\right\} .
\end{aligned}
$$

Recall that we have the following embedding for every $0 \leqslant t_{1}<t_{2}$ (see [12, Lemmas II.3.3, II.3.4]):

$$
W_{q}^{2,1}\left(Q_{t_{1}, t_{2}}\right) \hookrightarrow C_{\alpha}^{1,0}\left(\bar{Q}_{t_{1}, t_{2}}\right), \quad \forall 0 \leqslant \alpha<1-\frac{N+2}{q} .
$$

In the nondegenerate case, we have the following result.

Theorem 1.3. Suppose that b satisfies (b1) and

(b2') there exists $b_{0}>0$ such that $b(x) \geqslant b_{0}$ for a.e. $x \in \Omega$;

and that $u_{0}$ satisfies (H1) and $0 \leqslant u_{0} \leqslant 1$ for a.e. $x \in \Omega$. Then, in addition to the conclusions of Theorem 1.1, we have that

$$
u_{p} \rightarrow u \quad \text { strongly in } C_{\alpha}^{1,0}\left(\bar{Q}_{t_{1}, t_{2}}\right) \text {, weakly in } W_{q}^{2,1}\left(Q_{t_{1}, t_{2}}\right) \text { as } p \rightarrow+\infty \text {, }
$$

for every $\alpha \in(0,1), q \geqslant 1$ and $0<t_{1}<t_{2}$. Moreover, $u$ is the unique solution of

$$
\partial_{t} u-\Delta u=a u \chi_{\{u<1\}} \text { in } Q, \quad u(0)=u_{0}, \quad\|u\|_{\infty} \leqslant 1 .
$$

In this case, as $t \rightarrow+\infty$, we also obtain a convergence result for the coincidence sets $\{u(x, t)=1\}$.

Theorem 1.4. Suppose that b satisfies (b1)-(b2') and take $u_{0}$ satisfying (H1) and $0 \leqslant u_{0} \leqslant 1$ for a.e. $x \in \Omega$. Fix $a>\lambda_{1}(\Omega)$ and let $u$ be the unique solution of (8) and take $w$ the unique solution of (3). Then, as $t \rightarrow+\infty$,

$$
u(t) \rightarrow w \quad \text { strongly in } H_{0}^{1}(\Omega) \cap H^{2}(\Omega),
$$

and

$$
\chi_{\{u=1\}}(t) \rightarrow \chi_{\{w=1\}} \quad \text { strongly in } L^{q}(\Omega) \forall q \geqslant 1 .
$$

The structure of this paper is as follows: in Section 2 we prove Theorem 1.1 while in Section 3 Theorem 1.3 is treated. Finally, in Section 4 we show use the strong convergence up to the boundary of $\Omega$ obtained in the latter theorem to prove Theorem 1.4, and afterwards we use it combined with a subsolution argument to prove Theorem 1.2

We end this introduction by pointing out some other works concerning this type of asymptotic limit. The generalization of [5] for the $p$-Laplacian case was performed in [1]. In [1, 2], elliptic problems of type

$$
-\Delta u+f(x, u)|f(x, u)|^{p}=g(x)
$$


are treated, while in the works by Grossi et al [9, 10, and Bonheure and Serra [3], the authors dealt with the asymptotics study as $p \rightarrow+\infty$ of problems of type

$$
-\Delta u+V(|x|) u=u^{p}
$$

in a ball or annulus both with Neumann and Dirichlet boundary conditions.

\section{The general case: Proof of Theorem 1.1}

To make the presentation more structured, we split our proof in several lemmas. We start by showing a very simple comparison principle, which is an easy consequence of the monotonicity of the operator $u \mapsto|u|^{p-1} u$.

Lemma 2.1. Suppose that $u$ is a solution of (1) and take $v$ a supersolution satisfying

$$
\left\{\begin{array}{l}
\partial_{t} v-\Delta v \geqslant a v-b(x) v^{p} \text { in } Q_{T} \\
v(0)=v_{0},\left.\quad v(t)\right|_{\partial \Omega}=0
\end{array} \quad \text { with } u_{0} \leqslant v_{0} .\right.
$$

Then $u(x, t) \leqslant v(x, t)$ a.e.. On the other hand if $v$ is a subsolution satisfying

$$
\left\{\begin{array}{l}
\partial_{t} v-\Delta v \leqslant a v-b(x) v^{p} \text { in } Q_{T} \\
v(0)=v_{0},\left.\quad v(t)\right|_{\partial \Omega}=0
\end{array} \quad \text { with } v_{0} \leqslant u_{0},\right.
$$

then $v(x, t) \leqslant u(x, t)$.

Proof. The proof is quite standard, but we include it here only for the sake of completeness. In the case where $v$ is a supersolution, we have

$$
\partial_{t}(u-v)-\Delta(u-v)+b(x)\left(u^{p}-v^{p}\right) \leqslant a(u-v) .
$$

Multiplying this by $(u(t)-v(t))^{+}$, we obtain

$$
\begin{aligned}
\frac{1}{2} \frac{d}{d t} \int_{\Omega}[(u(t) & \left.-v(t))^{+}\right]^{2} d x \\
+ & \int_{\Omega}\left|\nabla(u(t)-v(t))^{+}\right|^{2} d x \\
& +\int_{\Omega} b(x)\left(u^{p}(t)-v^{p}(t)\right)(u(t)-v(t))^{+} d x \leqslant a \int_{\Omega}\left[(u(t)-v(t))^{+}\right]^{2} d x
\end{aligned}
$$

As $b(x)\left(u^{p}-v^{p}\right)(u-v)^{+} \geqslant 0$, we have

$$
\frac{d}{d t} \int_{\Omega}\left[(u(t)-v(t))^{+}\right]^{2} d x \leqslant 2 a \int_{\Omega}\left[(u(t)-v(t))^{+}\right]^{2} d x,
$$

whence

$$
\int_{\Omega}\left[(u(t)-v(t))^{+}\right]^{2} d x \leqslant e^{2 a t} \int_{\Omega}\left[\left(u_{0}-v_{0}\right)^{+}\right]^{2} d x=0 .
$$

The proof of the result for the subsolution case is analogous.

Next we show some uniform bounds in $p$.

Lemma 2.2. Given $T>0$ there exists $M=M(T)>0$ such that $\left\|u_{p}\right\|_{L^{\infty}\left(Q_{T}\right)} \leqslant M$ for all $p>1$.

Proof. Take $\psi \geqslant 0$ the unique solution of

$$
\left\{\begin{array}{l}
\partial_{t} \psi-\Delta \psi=a \psi \quad \text { in } Q_{T} \\
\psi(0)=u_{0},\left.\quad u(t)\right|_{\partial \Omega}=0
\end{array}\right.
$$

Then

$$
\partial_{t} \psi-\Delta \psi-a \psi+b(x) \psi^{p} \geqslant \partial_{t} \psi-\Delta \psi-a \psi=0,
$$


hence $\psi$ is a supersolution and from Lemma 2.1 we have that $0 \leqslant u_{p} \leqslant \psi$. In particular,

$$
\left\|u_{p}\right\|_{L^{\infty}\left(Q_{T}\right)} \leqslant\|\psi\|_{L^{\infty}\left(Q_{T}\right)}<+\infty, \quad\left(\text { as } u_{0} \in L^{\infty}(\Omega)\right),
$$

which proves the result.

Lemma 2.3. Given $T>0$, the sequence $\left\{u_{p}\right\}_{p}$ is bounded in $H^{1}\left(0, T ; L^{2}(\Omega)\right) \cap$ $L^{\infty}\left(0, T ; H_{0}^{1}(\Omega)\right)$. Thus there exists $u \in H^{1}\left(0, T ; L^{2}(\Omega)\right) \cap L^{\infty}\left(0, T ; H_{0}^{1}(\Omega)\right)$ such that

$$
\begin{aligned}
& u_{p} \rightarrow u \quad \text { strongly in } L^{2}\left(Q_{T}\right), \text { weakly in } L^{2}\left(0, T ; H_{0}^{1}(\Omega)\right), \\
& \partial_{t} u_{p} \rightarrow \partial_{t} u \quad \text { weakly in } L^{2}\left(Q_{T}\right) .
\end{aligned}
$$

Moreover, the exists $C=C(T)>0$ such that

$$
\iint_{Q_{T}} b(x) u_{p}^{p+1} d x d t \leqslant C, \quad \forall p>1 .
$$

Proof. Multiplying equation (11) by $u_{p}$ and integrating in $\Omega$,

$$
\frac{1}{2} \frac{d}{d t} \int_{\Omega} u_{p}^{2}(t) d x+\int_{\Omega}\left|\nabla u_{p}(t)\right|^{2} d x=a \int_{\Omega} u_{p}^{2}(t) d x-\int_{\Omega} b(x) u_{p}^{p+1}(t) d x ;
$$

integrating now between 0 and $t$,

$$
\begin{aligned}
\frac{1}{2} \int_{\Omega} u_{p}^{2}(t) d x+\int_{0}^{t} \| & \nabla u_{p}(\xi) \|_{2}^{2} d \xi+\iint_{Q_{t}} b(x) u_{p}^{p+1} d x d t \\
& \leqslant \frac{1}{2} \int_{\Omega} u_{0}^{2} d x+a \iint_{Q_{t}} u_{p}^{2} d x d t \leqslant \frac{1}{2}\left\|u_{0}\right\|_{2}^{2}+a t|\Omega|(M(t))^{2},
\end{aligned}
$$

and hence

$$
\forall T>0, \quad\left\{u_{p}\right\}_{p} \text { is bounded in } L^{2}\left(Q_{T}\right), \text { and (10) holds. }
$$

Now using $\partial_{t} u_{p}$ as test function $\left(u_{p}=0\right.$ on $\partial \Omega$ for all $t>0$, thus $\partial_{t} u_{p}(t) \in H_{0}^{1}(\Omega)$ for a.e. $t>0)$ gives

$$
\int_{\Omega}\left(\partial_{t} u_{p}\right)^{2} d x+\frac{1}{2} \frac{d}{d t} \int_{\Omega}\left|\nabla u_{p}(t)\right|^{2} d x=\frac{a}{2} \frac{d}{d t} \int_{\Omega} u_{p}^{2}(t) d x-\frac{d}{d t} \int_{\Omega} b(x) \frac{u_{p}^{p+1}(t)}{p+1} d x
$$

and again after an integration

$$
\begin{aligned}
& \text { (11) } \iint_{Q_{t}}\left(\partial_{t} u_{p}\right)^{2} d x d t+\frac{1}{2} \int_{\Omega}\left|\nabla u_{p}(t)\right|^{2} d x+\frac{a}{2} \int_{\Omega} u_{0}^{2} d x+\int_{\Omega} b(x) \frac{u_{p}^{p+1}(t)}{p+1} d x \\
& =\frac{1}{2} \int_{\Omega}\left|\nabla u_{0}\right|^{2} d x+\int_{\Omega} b(x) \frac{u_{0}^{p+1}}{p+1} d x+\frac{a}{2} \int_{\Omega} u_{p}^{2}(t) d x \leqslant \frac{1}{2}\left\|\nabla u_{0}\right\|_{2}^{2}+\frac{b_{\infty}|\Omega|}{p+1}+\frac{a}{2} M^{2}|\Omega|,
\end{aligned}
$$

where we have used the fact that $0 \leqslant u_{0} \leqslant 1$ whenever $b(x) \neq 0$, together with the previous lemma.

The proofs of the following two results are inspired by similar computations made in 1, 2.

Lemma 2.4. We have $u(t) \in \mathbb{K}_{0}$ for a.e. $t>0$. 
Proof. Let $\Omega^{\prime} \Subset \Omega \backslash \Omega_{0}$ and take $Q_{T}^{\prime}:=\Omega^{\prime} \times(0, T)$. Given $m>1$, we will show that $\left|\left\{(x, t) \in Q_{T}^{\prime}: u>m\right\}\right|=0$. Denote by $\underline{\mathrm{b}}$ the infimum of $b(x)$ over $\overline{\Omega^{\prime}}$, which is positive by (b2). Recalling (10), we deduce the existence of $C>0$ such that

$$
\begin{aligned}
0 \leqslant \iint_{\left\{u_{p}>m\right\} \cap Q_{T}^{\prime}} \underline{b} u_{p} d x d t & \leqslant \frac{1}{m^{p}} \iint_{\left\{u_{p}>m\right\} \cap Q_{T}^{\prime}} b(x) u_{p}^{p+1} d x d t \\
& \leqslant \frac{1}{m^{p}} \iint_{Q_{T}} b(x) u_{p}^{p+1} d x d t \leqslant \frac{C}{m^{p}}
\end{aligned}
$$

hence, as $m>1$ and $\underline{b}>0$,

$$
\lim _{p \rightarrow+\infty} \iint_{\left\{u_{p}>m\right\} \cap Q_{T}^{\prime}} u_{p} d x d t=0
$$

Now observe that

$$
\begin{aligned}
0 & =\lim _{p \rightarrow+\infty} \iint_{\left\{u_{p}>m\right\} \cap Q_{T}^{\prime}} u_{p} d x d t \\
& =\lim _{p \rightarrow+\infty}\left(\int_{0}^{T} \int_{\Omega^{\prime}} u_{p} \chi_{\left\{u_{p}>m\right\}} \chi_{\{u>m\}} d x d t+\int_{0}^{T} \int_{\Omega^{\prime}} u_{p} \chi_{\left\{u_{p}>m\right\}} \chi_{\{u \leqslant m\}} d x d t\right) \\
& \geqslant \lim _{p \rightarrow+\infty} \int_{0}^{T} \int_{\Omega^{\prime}} u_{p} \chi_{\left\{u_{p}>m\right\}} \chi_{\{u>m\}} d x d t
\end{aligned}
$$

As $u_{p} \chi_{\left\{u_{p}>m\right\}} \chi_{\{u>m\}} \rightarrow u \chi_{\{u>m\}}$ a.e. and $\left|u_{p} \chi_{\left\{u_{p}>m\right\}} \chi_{\{u>m\}}\right| \leqslant L$ on $Q_{T}$, then by the Lebesgue's dominated convergence Theorem we have

$$
\begin{aligned}
\lim _{p \rightarrow+\infty} \int_{0}^{T} \int_{\Omega^{\prime}} u_{p} \chi_{\left\{u_{p}>m\right\}} \chi_{\{u>m\}} d x d t & =\int_{0}^{T} \int_{\Omega^{\prime}} u \chi_{\{u>m\}} d x d t \\
& \geqslant m\left|\left\{(t, x) \in Q_{T}^{\prime}: u(t, x)>m\right\}\right| \geqslant 0
\end{aligned}
$$

and hence $\left|\left\{(x, t) \in Q_{T}^{\prime}: u(x, t)>m\right\}\right|=0 \quad$ whenever $m>1$.

Lemma 2.5. Let $u$ be the limit provided by Lemma 2.3. Then, up to a subsequence,

$$
u_{p} \rightarrow u \quad \text { strongly in } L^{2}\left(0, T ; H_{0}^{1}(\Omega)\right) .
$$

Proof. Multiplying equation (11) by $u_{p}-u$ and integrating in $Q_{T}$,

$$
\begin{aligned}
\iint_{Q_{T}} \partial_{t} u_{p}\left(u_{p}-u\right) d x d t+\iint_{Q_{T}} \nabla u_{p} \cdot \nabla\left(u_{p}-u\right) d x d t & +\iint_{Q_{T}} b(x) u_{p}^{p}\left(u_{p}-u\right) d x d t \\
& =\iint_{Q_{T}} a u_{p}\left(u_{p}-u\right) d x d t
\end{aligned}
$$

which, after adding and subtracting $\iint_{Q_{T}} \nabla u \cdot \nabla\left(u_{p}-u\right) d x d t$, is equivalent to

$$
\begin{aligned}
\iint_{Q_{T}} \partial_{t} u_{p}\left(v-u_{p}\right) d x d t & +\iint_{Q_{T}}\left|\nabla\left(u_{p}-u\right)\right|^{2} d x d t+\iint_{Q_{T}} \nabla u \cdot \nabla\left(u_{p}-u\right) d x d t \\
& +\iint_{Q_{T}} b(x) u_{p}^{p}\left(u_{p}-u\right) d x d t=\iint_{Q_{T}} a u_{p}\left(u_{p}-u\right) d x d t
\end{aligned}
$$

By the convergences shown in Lemma 2.3. we have that the terms $\iint_{Q_{T}} \partial_{t} u_{p}\left(u_{p}-\right.$ $u) d x d t, \iint_{Q_{T}} \nabla u \cdot \nabla\left(u_{p}-u\right) d x d t$ and $\iint_{Q_{T}} a u_{p}\left(u_{p}-u\right) d x d t$ tend to zero as $p \rightarrow+\infty$. 
Finally, observe that

$$
\begin{aligned}
\iint_{Q_{T}} b(x) & u_{p}^{p}\left(u_{p}-u\right) d x d t=\iint_{\left\{u_{p} \leqslant u\right\}} b(x) u_{p}^{p}\left(u_{p}-u\right) d x d t \\
& +\iint_{\left\{u<u_{p}\right\}} b(x) u_{p}^{p}\left(u_{p}-u\right) d x d t \geqslant \iint_{\left\{0 \leqslant u_{p} \leqslant u\right\}} b(x) u_{p}^{p}\left(u_{p}-u\right) d x d t .
\end{aligned}
$$

As $u \leqslant 1$ a.e. in $Q_{T}^{\prime}=(0, T) \times \Omega \backslash \Omega_{0}$ (cf. Lemma 2.4), we have

$$
\begin{aligned}
\left|\iint_{\left\{0 \leqslant u_{p} \leqslant u\right\}} b(x) u_{p}^{p}\left(u_{p}-u\right) d x d t\right| & \leqslant \iint_{\left\{0 \leqslant u_{p} \leqslant u\right\} \cap Q_{T}^{\prime}} b(x) u^{p}\left|u_{p}-u\right| d x d t \\
& \leqslant \iint_{Q_{T}} b_{\infty}\left|u_{p}-u\right| d x d t \rightarrow 0,
\end{aligned}
$$

whence $\liminf \iint_{Q_{T}} b(x) u_{p}^{p}\left(u_{p}-u\right) d x d t \geqslant 0$. Thus

$$
\iint_{Q_{T}}\left|\nabla\left(u_{p}-u\right)\right|^{2} d x d t \rightarrow 0 \quad \text { as } p \rightarrow+\infty,
$$

and the result follows.

Proof of Theorem 1.1. 1. The convergences of $u_{p}$ to $u$ are a consequence of Lemmas 2.3 and 2.5. Let us then prove first of all that

$$
\iint_{Q_{T}} \partial_{t} u(v-u) d x d t+\iint_{Q_{T}} \nabla u \cdot \nabla(v-u) d x d t \geqslant \iint_{Q_{T}} a u(v-u) d x d t
$$

for every $v \in \widetilde{\mathbb{K}}_{0}$, where $\widetilde{\mathbb{K}}_{0}:=\left\{v \in L^{2}\left(0, T ; H_{0}^{1}(\Omega)\right): v(t) \in \mathbb{K}_{0}\right.$ for a.e. $\left.t \in(0, T)\right\}$. Fix $v \in \widetilde{\mathbb{K}}_{0}$ and take $0<\theta<1$. Multiplying (1) by $\theta v-u_{p}$ and integrating we have

$$
\begin{aligned}
\iint_{Q_{T}} \partial_{t} u_{p}\left(\theta v-u_{p}\right) d x d t+\iint_{Q_{T}} \nabla u_{p} \cdot \nabla\left(\theta v-u_{p}\right) d x d t & +\iint_{Q_{T}} b(x) u_{p}^{p}\left(\theta v-u_{p}\right) d x d t \\
& =\iint_{Q_{T}} a u_{p}\left(\theta v-u_{p}\right) d x d t
\end{aligned}
$$

By Lemmas 2.3 and 2.5 we have that

$$
\begin{aligned}
& \iint_{Q_{T}} \partial_{t} u_{p}\left(\theta v-u_{p}\right) d x d t \rightarrow \iint_{Q_{T}} \partial_{t} u(\theta v-u) d x d t \\
& \iint_{Q_{T}} \nabla u_{p} \cdot \nabla\left(\theta v-u_{p}\right) d x d t \rightarrow \iint_{Q_{T}} \nabla u \cdot \nabla(\theta v-u) d x d t, \\
& \iint_{Q_{T}} u_{p}\left(\theta v-u_{p}\right) d x d t \rightarrow \iint_{Q_{T}} u(\theta v-u) d x d t .
\end{aligned}
$$

As for the remaining term, as $b(x)=0$ a.e. in $\Omega_{0}$ and $v \leqslant 1$ a.e in $\Omega \backslash \Omega_{0} \times(0, T)$, we have

$$
\begin{aligned}
\iint_{Q_{T}} b(x) & u_{p}^{p}\left(\theta v-u_{p}\right) d x d t=\iint_{\left\{0 \leqslant u_{p} \leqslant \theta v\right\}} b(x) u_{p}^{p}\left(\theta v-u_{p}\right) d x d t \\
& +\iint_{\left\{\theta v<u_{p}\right\}} b(x) u_{p}^{p}\left(\theta v-u_{p}\right) d x d t \leqslant \iint_{Q_{T}^{\prime}} b(x) \theta^{p}\left|\theta v-u_{p}\right| d x d t \rightarrow 0
\end{aligned}
$$


as $p \rightarrow+\infty$, because $\theta<1$. Thus

$$
\iint_{Q_{T}} \partial_{t} u(\theta v-u) d x d t+\iint_{Q_{T}} \nabla u \cdot \nabla(\theta v-u) d x d t \geqslant \iint_{Q_{T}} a u(\theta v-u) d x d t
$$

and now we just have to make $\theta \rightarrow 1$.

2. Given $v \in \mathbb{K}_{0}$ and $\xi \in(0, T), h>0$, take

$$
\tilde{v}(t)=\left\{\begin{array}{cc}
v & \text { if } t \in[\xi, \xi+h] \\
u(t) & \text { if } t \notin[\xi, \xi+h]
\end{array}\right.
$$

Then $\tilde{v} \in \widetilde{\mathbb{K}}_{0}$ and from (12) we have that

$$
\int_{\xi}^{\xi+h} \int_{\Omega} \partial_{t} u(v-u) d x d t+\int_{\xi}^{\xi+h} \int_{\Omega} \nabla u \cdot \nabla(v-u) d x d t \geqslant \int_{\xi}^{\xi+h} \int_{\Omega} a u(v-u) d x d t .
$$

Multiplying this inequality by $1 / h$ and making $h \rightarrow 0$ we get (6), as wanted.

3. Finally, it is easy to show that problem (6) has a unique solution. In fact, taking $u_{1}$ and $u_{2}$ solutions of (6) with same initial data, we have

$$
\begin{aligned}
\int_{\Omega} \partial_{t}\left(u_{1}(t)-u_{2}(t)\right)\left(u_{2}(t)-u_{1}(t)\right)+ & \left.\nabla\left(u_{1}(t)-u_{2}(t)\right) \cdot \nabla\left(u_{2}(t)-u_{1}(t)\right)\right) d x \\
& \geqslant \int_{\Omega} a\left(u_{1}(t)-u_{2}(t)\right)\left(u_{2}(t)-u_{1}(t)\right) d x
\end{aligned}
$$

which is equivalent to

$$
\frac{1}{2} \frac{d}{d t} \int_{\Omega}\left(u_{1}(t)-u_{2}(t)\right)^{2} d x+\int_{\Omega}\left|\nabla\left(u_{1}(t)-u_{2}(t)\right)\right|^{2} d x \leqslant \int_{\Omega} a\left(u_{1}(t)-u_{2}(t)\right)^{2} d x .
$$

The fact that $u_{1}, u_{2}$ have the same initial data now implies that

$$
\int_{\Omega}\left(u_{1}(t)-u_{2}(t)\right)^{2}(t) d x \leqslant e^{2 a t} \int_{\Omega}\left(u_{0}-u_{0}\right) d x=0 .
$$

Hence $u_{p} \rightarrow u$ for the whole sequence $\left\{u_{p}\right\}_{p}$, not only for a subsequence.

\section{The non Degenerate Case: Proof of Theorem 1.3}

As stated, the results of the previous section are true even in the case where $\Omega_{0}=\emptyset$. Let us check that in the non degenerate case (b2') we have a stronger convergence as well as a more detailed characterization for the limit $u$ (cf. (8)). This is mainly due to the following powerful estimate.

Lemma 3.1. There exists a constant $M>0$ (independent of $p$ ) such that $\left\|u_{p}\right\|_{L^{\infty}(Q)}^{p-1} \leqslant$ $M$ for all $p>1$.

Proof. Let $b_{0}=\inf _{\Omega} b>0$ and take $M_{p}>0$ such that $a M_{p}-b_{0} M_{p}^{p}=0$. Observe that $a s-b_{0} s^{p} \leqslant 0$ for $s \geqslant M_{p}$. Take $N_{p}:=\max \left\{1, M_{p}\right\}$. Multiplying (1) by $\left(u_{p}(t)-N_{p}\right)^{+}$(recall that $u_{p}=0$ on $\partial \Omega$, whence $\left(u_{p}-N_{p}\right)^{+}=0$ on the boundary as well), we obtain

$$
\begin{aligned}
& \frac{1}{2} \frac{d}{d t} \int_{\Omega}\left(\left(u_{p}-N_{p}\right)^{+}\right)^{2} d x+\int_{\Omega}\left|\nabla\left(u_{p}-N_{p}\right)^{+}\right|^{2} d x=\int_{\Omega}\left(a u_{p}-b(x) u_{p}^{p}\right)\left(u_{p}-N_{p}\right)^{+} d x \\
& \leqslant \int_{\Omega}\left(a u_{p}-b_{0} u_{p}^{p}\right)\left(u_{p}-N_{p}\right)^{+} d x=\int_{\left\{u_{p} \geqslant N_{p}\right\}}\left(a u_{p}-b_{0} u_{p}^{p}\right)\left(u_{p}-N_{p}\right) d x \leqslant 0 .
\end{aligned}
$$


Thus

$$
\frac{d}{d t} \int_{\Omega}\left(\left(u_{p}-N_{p}\right)^{+}\right)^{2} \leqslant 0, \quad \text { and } \quad \int_{\Omega}\left(\left(u_{p}(t)-N_{p}\right)^{+}\right)^{2} d x \leqslant \int_{\Omega}\left(\left(u_{0}-N_{p}\right)^{+}\right)^{2} d x
$$

which is zero because $N_{p} \geqslant 1$. Then $0 \leqslant u_{p}(t, x) \leqslant \max \left\{1, M_{p}\right\}$ and the result now follows from the fact that $M_{p}=\left(a / b_{0}\right)^{1 /(p-1)}$.

Lemma 3.2. For each $t_{2}>t_{1}>0, q>1$ and $\alpha \in(0,1)$, the sequence $\left\{u_{p}\right\}_{p}$ is bounded in $W_{q}^{2,1}\left(Q_{t_{1}, t_{2}}\right)$ and in $C_{\alpha}^{1,0}\left(\bar{Q}_{t_{1}, t_{2}}\right)$. Thus

$u_{p} \rightarrow u$, weakly in $W_{q}^{2,1}\left(Q_{t_{1}, t_{2}}\right), \quad u_{p} \rightarrow u$ strongly in $C_{\alpha}^{1,0}\left(\bar{Q}_{t_{1}, t_{2}}\right), \forall \alpha \in(0,1)$.

Proof. From Lemma 3.1 we get that

$$
\left\|a u_{p}\right\|_{L^{\infty}(Q)} \leqslant C^{\prime} M^{1 /(p-1)} \leqslant C^{\prime \prime}, \quad \text { and } \quad\left\|b(x) u_{p}^{p}\right\|_{L^{\infty}(Q)} \leqslant b_{\infty} M^{\frac{p}{p-1}} \leqslant C^{\prime \prime \prime},
$$

hence

$$
\left\|\partial_{t} u_{p}-\Delta u_{p}\right\|_{L^{\infty}(Q)} \leqslant C \quad \forall p>1
$$

which, together with [12, IV. Theorems $9.1 \& 10.1$ ] (see also [13, Theorem $7.22 \&$ $7.32]$ ), implies that for every $q>1$, the sequence $\left\{u_{p}\right\}_{p}$ is bounded in $W_{q}^{2,1}\left(Q_{t_{1}, t_{2}}\right)$ independently of $p$. Thus we can use the embedding (7) to show that $\left\{u_{p}\right\}_{p}$ is bounded in $C_{\alpha}^{1,0}\left(\bar{Q}_{t_{1}, t_{2}}\right)$. As the embedding $C_{\alpha}^{1,0} \hookrightarrow C_{\alpha^{\prime}}^{1,0}$ is compact for all $\alpha>\alpha^{\prime}$, we have the conclusion.

Observe that by Theorem 1.1 the whole sequence $u_{p}$ already converges to $u$ in some spaces, and hence the convergence obtained in this lemma is also for the whole sequence, not only for a subsequence.

Remark 3.3. It was important to assume $\Omega$ smooth (say $\partial \Omega$ of class $C^{2}$ ) to get regularity up to $\partial \Omega$. This will be of crucial importance in the next section. Without such regularity assumption, we would obtain convergence in each set of type $\Omega^{\prime} \times\left(t_{1}, t_{2}\right)$ with $\Omega^{\prime} \Subset \Omega, 0<t_{1}<t_{2}$.

Now, in view of Theorem [1.3. we want to prove that in this case $u$ solves (8). By Lemma 3.1. we know that $\left\|u_{p}^{p-1}\right\|_{L^{\infty}(Q)} \leqslant M$ for all $p>1$. This implies the existence of $\psi \geqslant 0$ such that, for every $T>0$,

$$
u_{p}^{p-1} \rightarrow \psi \quad \text { weak-* in } L^{\infty}\left(Q_{T}\right), \text { weak in } L^{2}\left(Q_{T}\right) .
$$

Thus when we make $p \rightarrow+\infty$ in (1) we obtain that the limit $u$ satisfies

$$
\partial_{t} u-\Delta u=(a-\psi) u \text {. }
$$

Moreover, $\left\|u_{p}\right\|_{\infty} \leqslant M^{\frac{1}{p-1}} \rightarrow 1$ as $p \rightarrow \infty$, which implies, together with Lemma 3.2 , that $0 \leqslant u \leqslant 1$. The proof of Theorem 1.3 will be complete after the following lemmas.

Lemma 3.4. $\psi=0$ a.e. in the set $\{(t, x) \in Q: u(x, t)<1\}$. In particular, this implies that

$$
\partial_{t} u-\Delta u=a u \chi_{\{u<1\}} \quad \text { a.e. }(x, t) \in Q .
$$

Proof. Take $(x, t)$ such that $u(x, t)<1$. As $u_{p} \rightarrow u$ in $C_{\alpha}^{1,0}$ we can take $\delta>0$ such that $u_{p} \leqslant 1-\delta$ for large $p$. Then $0 \leqslant u_{p}^{p-1} \leqslant(1-\delta)^{p-1} \rightarrow 0$ as $p \rightarrow+\infty$, whence $\psi(x, t)=0$. Thus $\psi=0$ a.e. on $\{(x, t): u(t, x)<1\}$.

Finally, as $u \in W_{q}^{2,1}$ for every $q \geqslant 1$, we have $\partial_{t} u-\Delta u=0$ a.e. on $\{(x, t)$ : $u(x, t)=1\}$ and the proof is complete. 
Lemma 3.5. Let $w$ be a solution of (8). Then $w$ solves (6).

Proof. Multiply equation (8) by $v-w$ with $v \in \mathbb{K}$. Then we have

$$
\begin{aligned}
\int_{\Omega} \partial_{t} w(v-w) d x+ & \int_{\Omega} \nabla w \cdot \nabla(v-w) d x=a \int_{\Omega} w \chi_{\{w<1\}}(v-w) d x \\
& =a \int_{\Omega} w(v-w) d x-a \int_{\Omega}(v-1) d x \geqslant a \int_{\Omega} w(v-w) d x,
\end{aligned}
$$

since $v \leqslant 1$ in $\Omega$.

Proof of Theorem 1.3. The convergences $u_{p} \rightarrow u$ strongly in $C_{\alpha}^{1,0}\left(\bar{Q}_{t_{1}, t_{2}}\right)$ and weakly in $W_{q}^{2,1}\left(Q_{t_{1}, t_{2}}\right)$ for every $T>0$ are a consequence of Lemma 3.2. By Lemma 3.4. $u$ satisfies (8). Finally Lemma 3.5 and the uniqueness show for (6) imply the uniqueness of solution of (8).

\section{Asymptotic Behavior as $t \rightarrow \infty$. Proof of Theorem 1.4}

In this section we will study the asymptotic behavior of (6) as $t \rightarrow+\infty$. First we need to understand what happens in the nondegenerate case (b2'), and prove Theorem 1.4 for that, as we will see, the convergence up to the boundary proved in Lemma 3.2 will be crucial. Only afterwards will we be able to prove Theorem 1.2 .

4.1. Proof of Theorem 1.4. We start by showing that the time derivative of $u$ vanishes as $t \rightarrow+\infty$.

Proposition 4.1. $\left\|\partial_{t} u(t)\right\|_{L^{2}(\Omega)} \rightarrow 0$ as $t \rightarrow+\infty$.

In order to prove this proposition, we will show that $\left\|\partial u_{p}(t)\right\|_{L^{2}(\Omega)} \rightarrow 0$ as $t \rightarrow+\infty$, uniformly in $p>1$. To do so, we will use the following result from 16 , Lemma 6.2.1].

Lemma 4.2. Suppose that $y(t), h(t)$ are nonnegative continuous functions defined on $[0, \infty)$ and satisfy the following conditions:

$$
y^{\prime}(t) \leqslant A_{1} y^{2}+A_{2}+h(t), \quad \int_{0}^{\infty} y(t) d t \leqslant A_{3}, \quad \int_{0}^{\infty} h(t) d t \leqslant A_{4},
$$

for some constants $A_{1}, A_{2}, A_{3}, A_{4}>0$. Then

$$
\lim _{t \rightarrow+\infty} y(t)=0 .
$$

Moreover, this convergence is uniform for all y satisfying (13) with the same constants $A_{1}, A_{2}, A_{3}, A_{4}$. 1

With this in mind, we have:

Lemma 4.3. Let $u_{p}$ be the solution of (1) and $a>0$. Then

$$
\left\|\partial_{t} u_{p}(t)\right\|_{2} \rightarrow 0 \quad \text { as } t \rightarrow+\infty, \text { uniformly in } p>1 \text {. }
$$

\footnotetext{
${ }^{1}$ This uniformity is not stated in the original lemma, but a close look at the proof allows us to easily obtain that conclusion.
} 
JOSÉ FRANCISCO RODRIGUES AND HUGO TAVARES

Proof. Let us check that $y(t):=\left\|\partial_{t} u_{p}(t)\right\|_{2}^{2}$ satisfies the assumptions of Lemma 4.2 First of all, (11) implies that

$$
\int_{0}^{\infty}\left\|\partial_{t} u_{p}(t)\right\|_{2}^{2} d x \leqslant\left\|\nabla u_{0}\right\|_{2}^{2}+\frac{|\Omega|}{2}+\frac{a}{2} M^{2}|\Omega|
$$

(recall that in the nondegenerate case $\left\|u_{p}\right\|_{L^{\infty}\left(\Omega \times \mathbb{R}^{+}\right)}$is bounded uniformly in $p$, by Lemma 3.1. Differentiate equation (1) with respect to $t$ :

$$
\partial_{t}^{2} u_{p}-\Delta \partial_{t} u_{p}+p u_{p}^{p-1} \partial_{t} u_{p}=a \partial_{t} u_{p}
$$

and multiply it by $\partial_{t} u_{p}$ and integrate in $\Omega$, at each time $t$. We obtain

$$
\begin{aligned}
\frac{1}{2} \frac{d}{d t} \int_{\Omega}\left(\partial_{t} u_{p}(t)\right)^{2} d x+ & \int_{\Omega}\left|\nabla\left(\partial_{t} u_{p}(t)\right)\right|^{2} d x+p \int_{\Omega} u_{p}^{p-1}(t)\left(\partial_{t} u_{p}(t)\right)^{2} d x \\
& =a \int_{\Omega}\left(\partial_{t} u_{p}(t)\right)^{2} d x \leqslant \frac{a}{2}\left(\int_{\Omega}\left(\partial_{t} u_{p}(t)\right)^{2} d x\right)^{2}+\frac{a}{2} .
\end{aligned}
$$

Thus

$$
\frac{d}{d t}\left\|\partial_{t} u_{p}(t)\right\|_{2}^{2} \leqslant a\left\|\partial_{t} u_{p}\right\|_{2}^{4}+a
$$

So we can apply the previous lemma with $A_{1}=a, A_{2}=a, A_{3}=\left\|\nabla u_{0}\right\|_{2}^{2}+\frac{|\Omega|}{2}+$ $\frac{a}{2} M^{2}|\Omega|$, and $h(t) \equiv 0, A_{4}=0$.

Proof of Proposition 4.1. From the previous lemma we know that, given $\varepsilon>0$, there exists $\bar{t}, p_{0}$ such that

$$
\left\|\partial_{t} u_{p}(t)\right\|_{2}^{2} \leqslant \varepsilon, \quad \forall t \geqslant \bar{t}, \quad \forall p>p_{0} .
$$

Thus for every $\bar{t} \leqslant t_{1}<t_{2}$,

$$
\int_{t_{1}}^{t_{2}}\left\|\partial_{t} u_{p}(t)\right\|_{2}^{2} d t \leqslant \varepsilon\left(t_{2}-t_{1}\right) \quad \forall t \geqslant \bar{t}, \quad \forall p>p_{0} .
$$

As $\partial_{t} u_{p} \rightarrow \partial_{t} u$ weakly in $L^{2}\left(Q_{T}\right)$ for every $T>0$ (cf. Theorem 1.1), then taking the $\liminf$ as $p \rightarrow+\infty$, we get

$$
\int_{t_{1}}^{t_{2}}\left\|\partial_{t} u(t)\right\|_{2}^{2} d t \leqslant \varepsilon\left(t_{2}-t_{1}\right), \quad \text { and hence }\left\|\partial_{t} u(t)\right\|_{2}^{2} \leqslant \varepsilon, \quad \forall t \geqslant \bar{t},
$$

which gives the statement.

Proof of Theorem 1.4. Fix $a>\lambda_{1}(\Omega)$. By taking $v=0$ in (6) we obtain

$$
\int_{\Omega}|\nabla u(t)|^{2} d x \leqslant \int_{\Omega}\left(-\partial_{t} u(t) u(t)+a u^{2}\right) d x
$$

which implies that $\|u(t)\|_{H_{0}^{1}(\Omega)}$ is bounded for $t>0$. Therefore, up to a subsequence, we have $u(t) \rightarrow \bar{u}$ in $H_{0}^{1}(\Omega)$ as $t \rightarrow+\infty$. Given a subsequence $t_{n} \rightarrow+\infty$ such that $u\left(t_{n}\right) \rightarrow \bar{u}$, we know that

$\int_{\Omega} \partial_{t} u\left(t_{n}\right)\left(v-u\left(t_{n}\right)\right) d x+\int_{\Omega} \nabla u\left(t_{n}\right) \cdot \nabla\left(v-u\left(t_{n}\right)\right) d x \geqslant a \int_{\Omega} u\left(t_{n}\right)\left(v-u\left(t_{n}\right)\right) d x$,

for all $v \in \mathbb{K}$ which, together with Proposition 4.1 implies that, as $p \rightarrow+\infty$,

$$
\int_{\Omega} \nabla \bar{u} \cdot \nabla(v-\bar{u}) d x \geqslant \int_{\Omega} a \bar{u}(v-\bar{u}) d x \quad \forall v \in \mathbb{K}
$$


or, equivalently,

$$
-\Delta \bar{u}=a \bar{u} \chi_{\{\bar{u}<1\}} .
$$

(here we are using the equivalence between these two problems which was shown in 44 and was stated in the Introduction). Since $\|\bar{u}\|_{\infty} \leqslant 1$ and $a>\lambda_{1}(\Omega)$, in order to prove that $\bar{u}=w$ (the unique nontrivial solution of (3)) the only thing left to prove is that $\bar{u} \not \equiv 0$.

2. Let us then check that, for $a>\lambda_{1}, \bar{u} \not \equiv 0$. Fix any $\bar{t}>0$. By the maximum principle we have that $u(\bar{t}, x)>0$ in $\Omega$ and $\partial_{\nu} u(\bar{t}, x)<0$ on $\partial \Omega$. By the convergence in $C_{\alpha}^{1,0}$-spaces up to the boundary of $\Omega$ (cf. Theorem [1.3) we have that, for $p \geqslant \bar{p}$, $u_{p}(\bar{t}, x)>0$ in $\Omega$ and $\partial_{\nu} u_{p}(\bar{t}, x)<0$ on $\partial \Omega$. Let $\varphi_{1}$ be the first eigenfunction of the Laplacian in $H_{0}^{1}(\Omega)$ with $\varphi_{1}>0$ and $\left\|\varphi_{1}\right\|_{\infty}=1$. Then

$$
c \varphi_{1} \leqslant u_{p}(\bar{t}, x) \quad \forall x \in \Omega, \forall p \geqslant \bar{p}
$$

for sufficiently small $c$ (independent of $p$ ). Observe moreover that $\partial_{t}\left(c \varphi_{1}\right)-\Delta\left(c \varphi_{1}\right) \leqslant$ $a\left(c \varphi_{1}\right)-b(x)\left(c \varphi_{1}\right)^{p}$ if and only if

$$
b(x) c^{p-1} \varphi_{1}^{p-1} \leqslant\left(a-\lambda_{1}\right) .
$$

Take $\bar{c}>0$ such that (14) and (15) hold. Then $\bar{c} \varphi_{1}$ is a subsolution of (11) for sufficiently small $\bar{c}$, for each $p \geqslant \bar{p}$. Then by Lemma 2.1 we have that $u_{p}(t, x) \geqslant \bar{c} \varphi_{1}$ for every $t \geqslant \bar{t}$ and $p \geqslant \bar{p}$, and hence as $p \rightarrow \infty$ also $u(t, x) \geqslant \bar{c} \varphi_{1}(x)$ for every $x \in \Omega, t \geqslant \bar{t}$. Thus $\bar{u} \not \equiv 0$ and $\bar{u}=w$, the unique solution of (3). From the uniqueness we deduce in particular that $u(t) \rightarrow w$ in $H_{0}^{1}(\Omega)$ as $t \rightarrow \infty$, not only for some subsequence. As for the strong convergence, this is now easy to show since by taking the difference

$$
\partial_{t} u-\Delta(u(t)-w)=a u(t) \chi_{\{u<1\}}-a w \chi_{\{w<1\}}
$$

and multiplying it by $u(t)-w$, we get

$\int_{\Omega}|\nabla(u(t)-w)|^{2}=-\int_{\Omega} \partial_{t} u(t)(u(t)-w)+\left(a u(t) \chi_{\{u<1\}}-a w \chi_{\{w<1\}}\right)(u(t)-w) \rightarrow 0$ as $t \rightarrow \infty$ (recall that both $u(t)$ and $w$ are less than or equal to 1$)$. Thus $u(t) \rightarrow w$ strongly in $H_{0}^{1}(\Omega)$.

3. The convergence of the coincidence sets follows as in [15]. As $0 \leqslant \chi_{\{u=1\}}(t) \leqslant$ 1 , then there exists a function $0 \leqslant \chi^{*} \leqslant 1$ such that, up to a subsequence,

$$
\chi_{\{u=1\}}(t) \rightarrow \chi^{*} \quad \text { weak- } * \text { in } L^{\infty}(\Omega) \text {, as } t \rightarrow+\infty .
$$

Since $\chi_{\{u=1\}}(1-u)=0$ a.e, then also $\chi^{*}(1-w)=0$ a.e. and hence $\chi^{*}=0$ whenever $w<1$. Moreover, from the fact that $\partial_{t} u-\Delta u=a u\left(1-\chi_{\{u=1\}}\right)$ a.e. in $Q$ we deduce that $-\Delta w=a w\left(1-\chi^{*}\right)$. As $\Delta w=0$ a.e. on $\{w=1\}$ (in fact, $u \in W^{2, q}(\Omega)$ for every $q \geqslant 1)$, we conclude that $\chi^{*}=1$ on $\{w=1\}$, whence $\chi^{*}=\chi_{\{w=1\}}$. Since in general $L^{\infty}(\Omega)$ weak-* convergence of characteristic functions imply strong convergence in $L^{q}(\Omega)$ for every $q \geqslant 1$, we have proved (9). As a consequence, actually $u(t) \rightarrow w$ in $H^{2}$-norm.

4. For $a<\lambda_{1}(\Omega)$, the function 0 attracts all the solutions of (6) with nonnegative initial data; in fact, by taking $v=0$ in (6) we obtain

$$
\int_{\Omega}|\nabla u(t)|^{2} d x \leqslant a \int_{\Omega} u(t)^{2} d x-\int_{\Omega} \partial_{t} u(t) u(t) d x \leqslant \frac{a}{\lambda_{1}(\Omega)} \int_{\Omega}|\nabla u(t)|^{2} d x+\mathrm{o}(1)
$$

as $t \rightarrow+\infty$, and thus $\|u(t)\|_{H_{0}^{1}(\Omega)} \rightarrow 0$. 
4.2. Proof of Theorem 1.2, Fix $a \in\left(\lambda_{1}(\Omega), \lambda_{1}\left(\Omega_{0}\right)\right)$. In this case we have a stronger result than Lemma 3.1 having a uniform $L^{\infty}$ bound in $Q=\Omega \times \mathbb{R}^{+}$.

Lemma 4.4. For $a \in\left(\lambda_{1}(\Omega), \lambda_{1}\left(\Omega_{0}\right)\right)$, there exists $C>0$ such that $\|u\|_{L^{\infty}\left(\mathbb{R}^{+} \times \Omega\right)} \leqslant$ $C$ for all $p>1$.

Proof. Here we follow the line of the proof of Claim 1 in [5, p. 224], to which we refer for more details. Define $\Omega_{\delta}=\left\{x \in \mathbb{R}^{N}: \operatorname{dist}(x, \Omega)<\delta\right\}$. Since $a<\lambda_{1}\left(\Omega_{0}\right)$, there exists a small $\delta$ such that $a<\lambda_{1}\left(\Omega_{\delta}\right)$ (by continuity of the map $\Omega \mapsto \lambda_{1}(\Omega)$ ). Denoting by $\phi_{\delta}$ the first eigenfunction of $-\Delta$ in $H_{0}^{1}\left(\Omega_{\delta}\right)$ and $\psi$ any extension of $\left.\phi\right|_{\Omega_{\delta / 2}}$ to $\bar{\Omega}$ such that $\min _{\bar{\Omega}} \psi>0$, there exists $Q>0$ large enough such that

$$
-\Delta(Q \psi)-a Q \psi+b(x)(Q \psi) \geqslant 0 \quad \text { in } \Omega,
$$

and $u_{0} \leqslant Q \psi$ in $\Omega$. Thus $Q \psi$ is a supersolution of (11) for all $p>1$ and by Lemma 2.1 we have

$$
u_{p} \leqslant Q \psi \leqslant M \quad \text { for all }(t, x) \in Q \text {. }
$$

Proof of Theorem 1.2. 1. Fix $a \in\left(\lambda_{1}(\Omega), \lambda_{1}\left(\Omega_{0}\right)\right)$. Having proved Lemma 4.4. we can repeat the proof of Proposition 4.1 word by word and show that

$$
\left\|\partial_{t} u(t)\right\|_{L^{2}(\Omega)} \rightarrow 0 \quad \text { as } t \rightarrow+\infty .
$$

By making $v=0$ in (6), we obtain once again by Lemma 4.4 that $\|u(t)\|_{H_{0}^{1}(\Omega)}$ is bounded for $t>0$. Take $t_{n} \rightarrow+\infty$ such that $u\left(t_{n}\right) \rightarrow \bar{u}$ in $H_{0}^{1}(\Omega)$ for some $\bar{u} \in H_{0}^{1}(\Omega)$. Then $\bar{u} \in \mathbb{K}_{0}$ and

$$
\int_{\Omega} \nabla \bar{u} \cdot \nabla(v-\bar{u}) d x \geqslant a \int_{\Omega} \bar{u}(v-\bar{u}) d x, \quad \forall v \in \mathbb{K}_{0} .
$$

In 4, Dancer and Du have shown that (16) has a unique nontrivial nonnegative solution $w$. In order to prove that $\bar{u}=w$ and conclude the proof for this case, we just have to show that $\bar{u} \not \equiv 0$. This will be a consequence of Theorem 1.4 In fact, considering $\phi_{p}$ as the solution of

$$
\left\{\begin{array}{l}
\partial_{t} \phi_{p}-\Delta \phi_{p}=a \phi_{p}-\|b\|_{\infty} \phi_{p}^{p} \quad \text { in } Q_{T} \\
u(0)=v_{0},\left.\quad u(t)\right|_{\partial \Omega}=0
\end{array}\right.
$$

with $v_{0}:=\inf \left\{u_{0}, 1\right\}$, it is straightforward to see that $\phi_{p}$ is a subsolution of (11), and

$$
u_{p} \geqslant \phi_{p} \rightarrow w \quad \text { as } p \rightarrow+\infty,
$$

where $w \neq 0$ is the unique nontrivial solution of (3). This last statement is a consequence of Theorem 1.4 as $0 \leqslant v_{0} \leqslant 1$ a.e. in $\Omega$. Thus $\bar{u} \geqslant w \not \equiv 0$, which concludes the proof in this case.

2. If $a<\lambda_{1}(\Omega)$, the same reasoning as in the proof of Theorem 1.4 yields that $\|u(t)\|_{H_{0}^{1}(\Omega)} \rightarrow 0$. As for the case $a \geqslant \lambda_{1}\left(\Omega_{0}\right)$, if either $\|u(t)\|_{\infty}$ or $\|u(t)\|_{H_{0}^{1}(\Omega)}$ were bounded, it is clear from the proof of Proposition 4.1 that $\left\|\partial_{t} u(t)\right\|_{L^{2}(\Omega)} \rightarrow 0$. Repeating the reasoning of the previous step, we would obtain a nontrivial solution of (6) for $a \geqslant \lambda_{1}\left(\Omega_{0}\right)$, contradicting [4, Theorem 1.1].

Remark 4.5. As for the case $a=\lambda_{1}(\Omega)$, observe that $c \varphi_{1}$ is always a steady state solution of (8) for all $0<c<1$, where $\varphi_{1}$ denotes the first eigenfunction of $\left(-\Delta, H_{0}^{1}(\Omega)\right)$ with $\left\|\varphi_{1}\right\|_{\infty}=1$. Hence the long time limit of (6) in this case will depend on the initial condition $u_{0}$, and we are only able to conclude that given 
$t_{n} \rightarrow+\infty$ there exists a subsequence $\left\{t_{n_{k}}\right\}$ such that $u\left(t_{n_{k}}\right)$ converges to $c \varphi_{1}$ for some $c>0$.

Acknowledgements. The second author would like to thank Jesús Hernández and Pedro Freitas for useful discussions related with this paper.

\section{REFERENCES}

[1] L. Boccardo and F. Murat, Increase of power leads to bilateral problems, Composite Media and Homogenization Theory, G. Dal Maso and G. F. Dell'Antonio, eds., World Scientific, Singapore, 1995, 113-123.

[2] A. Dall'Aglio and L. Orsina, On the limit of some nonlinear elliptic equations involving increasing powers, Asymptotic Analysis 14 (1997), 49-71.

[3] D. Bonheure and E. Serra, Multiple positive radial solutions on annuli for nonlinear Neumann problems with large growth, NoDEA 18 (2011), 217-235.

[4] E. Dancer And Y. Du, On a free boundary problem arising from population biology, Indiana Univ. Math. J. 52 (2003), 51-67.

[5] E. Dancer, Y. Du And L. MA, Asymptotic behavior of positive solutions of some elliptic problems, Pacific Journal of Mathematics 210 (2003), 215-228.

[6] E. Dancer, Y. Du And L. Ma, A uniqueness theorem for a free boundary problem, Proc. Amer. Math. Soc. 134 (2006), 3223-3230.

[7] Y. Du AND Z. Guo, The degenerate logistic model and a singularly mixed boundary blow-up problem, Discrete and Continuous Dyn. Syst. 14 (2006), 1-29.

[8] J. M. Fraile, P. Koch Medina, J. López-Gómez and S. Merino, Elliptic eigenvalue problems and unbounded continua of positive solutions of a semilinear elliptic equation, J. Differential Equations, 127 (1996), 295-319.

[9] M. Grossi, Asymptotic behaviour of the Kazdan-Warner solution in the annulus, J. Differential Equations 223 (2006), 96-111.

[10] M. Grossi and B. Noris, Positive constrained minimizers for supercritical problems in the ball, Proc. Amer. Math. Soc. 140 (2012), 2141-2154.

[11] Z. Guo And L. MA, Asymptotic behavior of positive solutions of some quasilinear elliptic problems, J. London Math. Soc. 76 (2007), 419-437.

[12] O. Ladyzenskaja, V. Solonnikov and N. Uralceva, Linear and quasi-linear equations of parabolic type, American Mathematical Society, 1988.

[13] G. Lieberman, Second order parabolic differential equations, World Scientific, 1996.

[14] J. L. Lions, Quelques méthodes de résolution des problémes aux limites non linéaires, Gauthier-Villars, Paris, 1969.

[15] J.F. Rodrigues, On a class of parabolic unilateral problems, Nonlinear Anal. 10 (1986), $1357-1366$.

[16] S. Zheng, Nonlinear Evolutions Equations, Chapman \& Hall/CRC Monographs and Surveys in Pure and Applied Mathematics, 133, Chapmam \& Hall/CRC, Boca Raton, FL, 2004.

Universidade de Lisboa/CMaf Av. Prof. Gama Pinto 2 1649-003 Lisboa, Portugal

E-mail address: rodrigue@ptmat.fc.ul.pt

Universidade de Lisboa/CMAf Av. Prof. Gama Pinto 2 1649-003 Lisboa, Portugal

E-mail address: htavares@ptmat.fc.ul.pt 